\title{
Percepção da doença: uma avaliação a ser realizada pelos enfermeiros
}

\author{
Illness perception: an evaluation to be conducted by nurses
}

Percepción de la enfermedad: una evaluación a ser realizada por los enfermeros

\author{
Paloma Ferrer Gomez', Maria Gaby Rivero de Gutiérrez", Rita Simone Lopes Moreira' \\ ' Universidade Federal de São Paulo, Escola Paulista de Enfermagem, \\ Residência em Enfermagem Cardiovascular. São Paulo-SP, Brasil. \\ " Universidade Federal de São Paulo, Escola Paulista de Enfermagem, \\ Curso de Graduação em Enfermagem. São Paulo-SP, Brasil.
}

Submissão: 31-03-2011 Aprovação: 12-10-2011

\section{RESUMO}

Percepção da doença é a maneira como os indivíduos entendem e lidam com sua patologia. O objetivo deste estudo foi identificar, na literatura, artigos desenvolvidos por enfermeiros a respeito da percepção da doença e os instrumentos disponíveis para a avaliação da mesma. Foi realizada uma revisão integrativa da literatura, que possibilitou a identificação de nove artigos que relacionavam a percepção da doença à Educação em saúde, Enfrentamento, Adesão ao tratamento e Qualidade de vida. Também foram identificadas formas de avaliação deste fenômeno. Concluiu-se que os enfermeiros devem avaliar periodicamente a percepção da doença por meio de instrumentos validados e, preferencialmente, em conjunto com o uso dos diagnósticos de enfermagem descritos pela NANDA Internacional (NANDA-I).

Descritores: Avaliação; Percepção da doença; Enfermagem.

\section{ABSTRACT}

Illness perception is understood as the way individuals understand and cope with their disease. The objective of this paper was to identify articles in literature developed by nurses on disease perception, and the instruments available to assess it. An integrative literature review was performed, which identified nine papers that related to the perception of the disease with Health Education, Coping, Treatment adherence and quality of life. Ways of assessing this phenomenon were also identified. It was concluded that nurses should periodically assess the perception of illness by validated instruments, along with the use of nursing diagnoses reported by NANDA International (NANDA-I).

Key words: Evaluation; Illness perception; Nursing.

\section{RESUMEN}

Percepción de la enfermedad es la forma como los individuos entienden y enfrentan con su patología. El objetivo de este estudio fue identificar, en la literatura, artículos desarrollados por enfermeras sobre la percepción de la enfermedad y los instrumentos disponibles para su evaluación. Se realizó una revisión integradora de la literatura, que posibilitó la identificación de nueve artículos que relacionaban la percepción de la enfermedad con la Educación para la salud, Afrontamiento, Adherencia al tratamiento y la Calidad de vida. También se identificaron las formas de evaluar este fenómeno. Se concluyó que las enfermeras deben evaluar periódicamente la percepción de la enfermedad por medio de instrumentos validados, de preferencia, en conjunto con el uso de los diagnósticos de enfermería reportados por NANDA Internacional (NANDA-I).

Palabras clave: Evaluación; Percepción de la Enfermedad; Enfermería. 


\section{INTRODUÇÃO}

O aparecimento de uma doença dá início a uma série de eventos fisiológicos, psíquicos, emocionais e sociais, que podem variar de paciente para paciente, mesmo que estes se encontrem em situações muito parecidas.

A percepção da doença é definida como a forma que os indivíduos compreendem diversos aspectos relacionados à saúde e a doença levando em consideração suas experiências individuais e coletivas ${ }^{(1)}$. Além disso, a percepção da doença inclui a informação que o indivíduo possui a respeito de sua patologia, bem como seus sintomas, potenciais causas, provável duração, evolução no tempo e potenciais consequências. Quando o indivíduo vivencia algum sintoma, inicia-se um processo de cognição no qual a pessoa passa a comparar este sintoma com o modelo que possui sobre a doença.

O modelo teórico de percepção da doença pode estar baseado no modelo dinâmico do comportamento do indivíduo, sendo esta percepção influenciada pelas crenças individuais baseadas em experiências vividas antes do aparecimento da doença e pelo processo que conduz ao comportamento da saú$\mathrm{de}^{(2)}$. O comportamento de saúde por sua vez é o resultado de um processo interativo pelo qual o indivíduo soma as informações de estímulos externos e internos, que dão sentido às suas experiências e reações emocionais em função de sua doença.

No Brasil, a avaliação da percepção que o paciente possui a respeito da doença realizada de forma estruturada, por meio de instrumentos específicos, não é algo tão presente no cotidiano dos profissionais de saúde, especialmente entre os enfermeiros, embora essa avaliação seja feita pelos mesmos de forma subjetiva.

Conhecer como o enfermeiro avalia a percepção da doença pelo paciente poderá contribuir para que este aspecto seja investigado de forma estruturada, de modo a permitir a identificação de diagnósticos de enfermagem sobre o mesmo e possibilitar o planejamento de intervenções que auxiliem o paciente
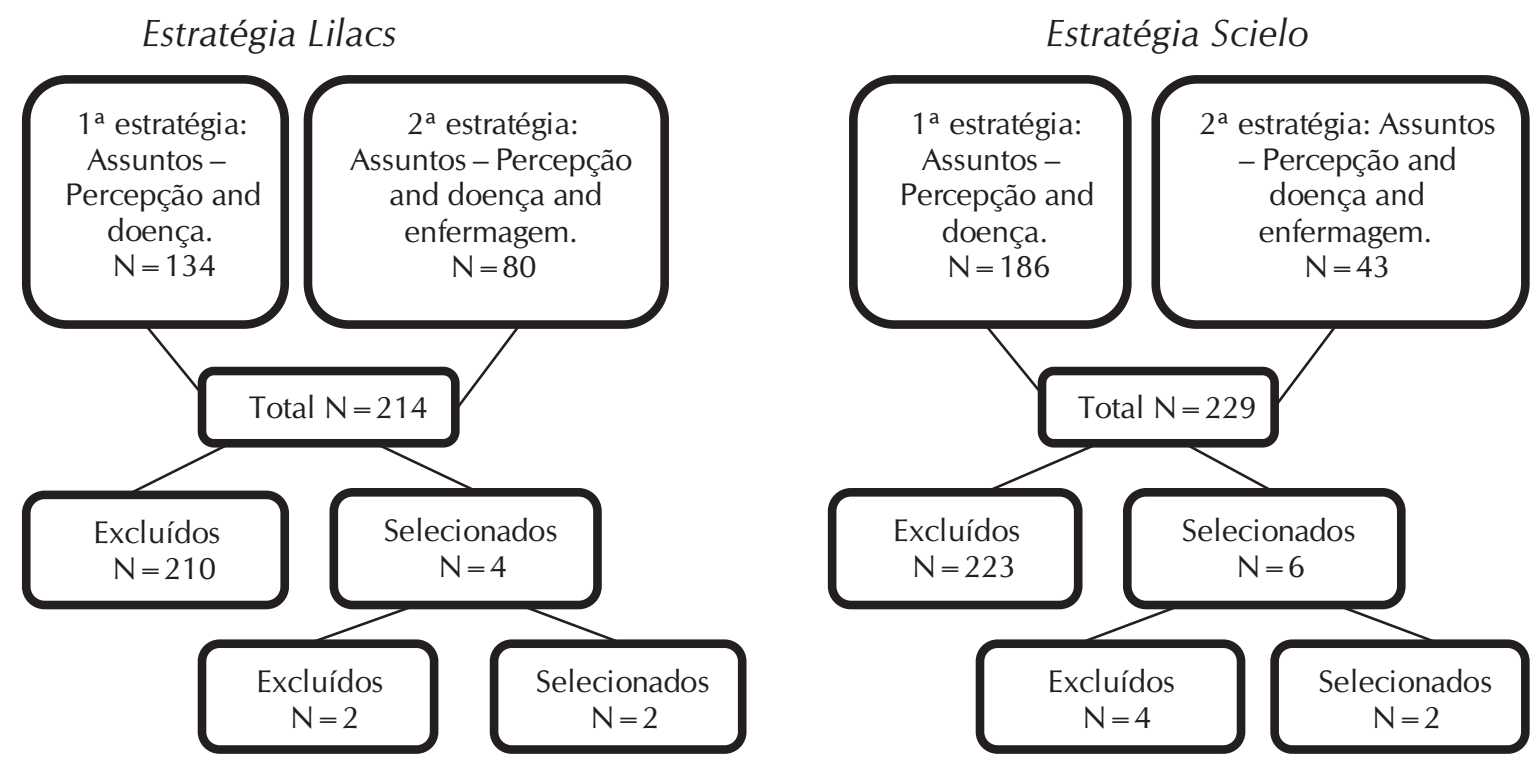

Estratégia Medline

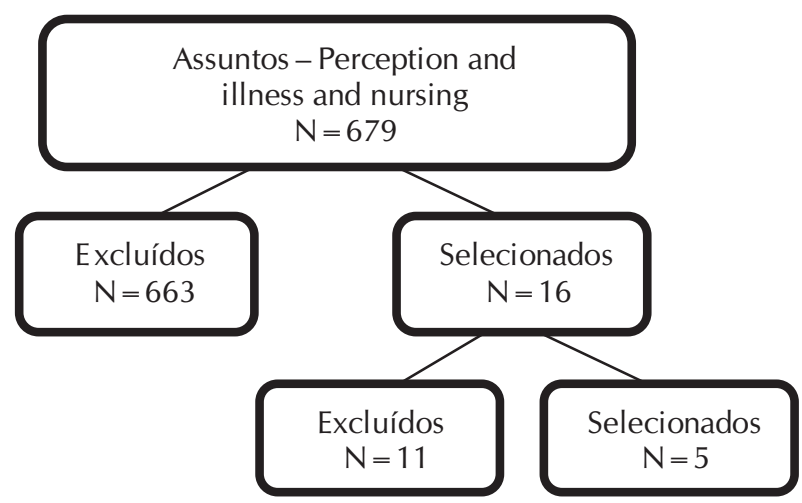

Figura 1 - Fluxograma de seleção de artigos 
na busca de estratégias para a convivência com sua patologia.

Tendo em vista os aspectos abordados, objetivou-se, com este estudo, identificar, na literatura, estudos realizados por enfermeiros a respeito da percepção da doença e descrever os instrumentos de avaliação por eles utilizados.

\section{MÉTODO}

Trata-se de uma revisão integrativa de literatura, desenvolvida com base nos passos propostos por Ganong. Foram elaboradas as seguintes perguntas de estudo: Quais os aspectos abordados nos estudos sobre a percepção da doença em adultos? Como os enfermeiros avaliam a percepção da doença por parte de pacientes adultos?.

Foram selecionados artigos indexados nas bases de dados LILACS, SCIELO e MEDLINE, utilizando os descritores "percepção", "doença" e "enfermagem", com o seguinte filtro: ("perception"[MeSH Terms] OR "perception"[All Fields]) AND illness[All Fields] AND ("nursing"[Subheading] OR "nursing"[All Fields] OR "nursing"[MeSH Terms] OR "nursing"[All Fields].

A busca por artigos se deu no período de março de 2010 a fevereiro de 2011. A revisão abrangeu o período de 2001 a 2011, incluindo artigos nas línguas portuguesa e inglesa que descreviam a percepção sobre a doença, os aspectos relacionados à mesma, bem como os instrumentos utilizados. Os artigos foram incluídos após análise de título e resumo.

De acordo com os critérios, foram excluídos os estudos que não abordavam a temática escolhida tais como: artigos com foco nos cuidadores, percepção da família, estudos que abordavam crianças ou adolescentes e estudantes de enfermagem.

Foram selecionados 26 artigos, sendo que nove atenderam os critérios de inclusão.

Os artigos incluídos na amostra foram lidos na íntegra e seu conteúdo analisado de acordo com a técnica descrita por Sampieri, que consiste em avaliar cada estudo a partir de uma unidade de análise (tema, item, caráter e tempo-espaço) e a partir daí, categorizar os estudos, agrupando-os de acordo com os dados encontrados em cada um ${ }^{(3)}$.

No presente estudo, a unidade de análise utilizada foi o tema e em seguida, os estudos foram categorizados de acordo com os aspectos relacionados à percepção da doença identificados na discussão de cada um deles.

\section{Resultados}

Apresentam-se as características da pesquisa primária, tendo como base a análise dos nove artigos selecionados. As informações obtidas foram registradas da seguinte maneira: título do artigo, número da referência, ano, objetivo, método, utilização de instrumentos para avaliação da percepção da doença e aspectos abordados em relação a essa percepção.

Os nove artigos analisados foram realizados por enfermeiros, e a maioria deles (6-66,6\%), publicados em periódicos de enfermagem, no período de 2008 e 2011. A abordagem adotada em sete $(77,8 \%)$ dos estudos foi a descritiva quantitativa e em dois $(22,2 \%)$ foi a qualitativa. Quanto ao delineamento utilizado, oito $(88,9 \%)$ são do tipo transversal e um $(11,1 \%)$ longitudinal. No que diz respeito à utilização de instrumentos para a avaliação da percepção a respeito da doença, quatro $(44,4 \%)$ utilizaram algum instrumento validado para essa avaliação e cinco $(55,6 \%)$ realizaram a avaliação por meio de entrevistas semi-estruturadas.

Com base na análise de conteúdo, foi possível identificar e categorizar os estudos de acordo com os aspectos abordados sobre a percepção da doença. O conteúdo abordado nos artigos foi categorizado em Educação em saúde, Enfrentamento, Adesão ao tratamento e Qualidade de vida. A maioria dos artigos abordou mais de uma categoria, sendo que oito deles discorreram sobre Educação em saúde; seis sobre Adesão ao tratamento; cinco sobre Enfrentamento, e quatro sobre Qualidade de vida. As categorias nas quais cada artigo foi incluído podem ser vistas no Quadro 1.

\section{Educação em saúde}

A educação em saúde representa uma estratégia fundamental no processo de formação de comportamentos que promovam ou mantenham uma boa saúde. Este processo deve ser capaz de criar condições para a construção de um conceito de saúde e doença, que leve em conta as condições de vida de cada indivíduo e que contribua, não só para despertar o sentimento de que é possível transformar a realidade, mas também conscientizar de que a saúde é um direito de todos ${ }^{(13)}$.

Enfermeiros capacitados são capazes de acompanhar a trajetória e a evolução da doença, traçar um plano educacional junto ao paciente e assim, promover a reflexão sobre a percepção da doença por parte do paciente e, consequentemente, contribuir para a melhora de sua qualidade de vida ${ }^{(12)}$.

O conhecimento da enfermagem a respeito da percepção que o paciente possui sobre sua doença permite aos profissionais a individualização de estratégias de educação e cuidado prestado $^{(7,9)}$.

A educação e a intervenção por parte da enfermagem é capaz de gerar maior adesão ao tratamento e promoção à saúde. Com uma assistência especificamente orientada a cada tipo de paciente, é possível obter bons resultados em relação à severidade da doença e qualidade de vida ${ }^{(5,11,12)}$.

Cabe aos profissionais de saúde, em especial ao enfermeiro, dialogar com o paciente sobre a sua patologia, dúvidas, tratamento e consequências da não adesão a este, para que juntos possam trabalhar e obter bons resultados ${ }^{(8,10)}$.

Apoiar o paciente a refletir como percebe e controla sua doença pode ser uma estratégia primária de enfermagem nos programas de reabilitação o que por sua vez, pode facilitar o comportamento de saúde, diminuir as experiências desconfortantes dos efeitos colaterais e melhorar a qualidade de vida ${ }^{(5,6,8)}$.

\section{Adesão ao tratamento}

Adesão ao tratamento é um fenômeno sujeito a influência de múltiplos fatores que afetam diretamente o paciente e determinam o comportamento deste em relação às recomendações referentes ao tratamento de sua doença. Esses fatores, por sua vez, estão relacionados às condições sócio demográficas, à doença, à terapêutica, à relação entre os profissionais 


\begin{tabular}{|c|c|c|c|c|c|}
\hline Título & Ano & Objetivo & Método & $\begin{array}{l}\text { Instrumento } \\
\text { utilizado }\end{array}$ & Aspectos abordados \\
\hline $\begin{array}{l}\text { Illness perceptions and adherence } \\
\text { to therapeutic regimens among } \\
\text { patients with hypertension: A } \\
\text { structural modeling approach. }{ }^{(4)}\end{array}$ & 2011 & $\begin{array}{l}\text { Analisar a relação entre percepção } \\
\text { da doença e adesão à medicação } \\
\text { prescrita e recomendações de auto- } \\
\text { gestão em pacientes hipertensos. }\end{array}$ & $\begin{array}{l}\text { Estudo } \\
\text { descritivo e } \\
\text { transversal }\end{array}$ & IPQ -R & $\begin{array}{l}\text { Adesão ao tratamento } \\
\text { Enfrentamento }\end{array}$ \\
\hline $\begin{array}{l}\text { Illness perception among Chinese } \\
\text { patients with acute myocardial } \\
\text { infarction. }{ }^{(5)}\end{array}$ & 2010 & $\begin{array}{l}\text { Explorar a percepção da doença } \\
\text { e seus preditores entre pacientes } \\
\text { chineses com infarto do miocárdio. }\end{array}$ & $\begin{array}{l}\text { Estudo } \\
\text { descritivo e } \\
\text { transversal }\end{array}$ & IPQ -R & $\begin{array}{l}\text { Educação em saúde } \\
\text { Enfrentamento }\end{array}$ \\
\hline $\begin{array}{l}\text { Illness Perceptions After } \\
\text { Myocardial Infarction: Relations to } \\
\text { Fatigue, Emotional Distress, and } \\
\text { Health-Related Quality of Life. }{ }^{(6)}\end{array}$ & 2010 & $\begin{array}{l}\text { Analisar a percepção da doença } \\
\text { e sua relação com a qualidade } \\
\text { de vida relatada por pacientes } \\
\text { infartados. }\end{array}$ & $\begin{array}{l}\text { Estudo } \\
\text { descritivo e } \\
\text { longitudinal }\end{array}$ & IPQ & $\begin{array}{l}\text { Educação em saúde } \\
\text { Enfrentamento } \\
\text { Qualidade de vida }\end{array}$ \\
\hline $\begin{array}{l}\text { Patients' illness perception } \\
\text { four months after a myocardial } \\
\text { infarction. }^{(7)}\end{array}$ & 2008 & $\begin{array}{l}\text { Investigar a percepção da doença } \\
\text { em pacientes infartados há quatro } \\
\text { meses. }\end{array}$ & $\begin{array}{l}\text { Estudo } \\
\text { observacional } \\
\text { transversal }\end{array}$ & $\begin{array}{l}\text { Entrevista } \\
\text { semi- } \\
\text { estruturada }\end{array}$ & $\begin{array}{l}\text { Educação em saúde } \\
\text { Adesão ao tratamento } \\
\text { Enfrentamento }\end{array}$ \\
\hline $\begin{array}{l}\text { Paciente crônico frente ao adoecer } \\
\text { e a aderência ao tratamento. }{ }^{\left({ }^{8}\right)}\end{array}$ & 2008 & $\begin{array}{l}\text { Conhecer a percepção da doença } \\
\text { por pacientes crônicos e motivos } \\
\text { da não adesão ao tratamento. }\end{array}$ & $\begin{array}{l}\text { Estudo } \\
\text { descritivo } \\
\text { transversal }\end{array}$ & $\begin{array}{l}\text { Entrevista semi- } \\
\text { estruturada } \\
\text { e análise de } \\
\text { prontuário }\end{array}$ & $\begin{array}{l}\text { Educação em saúde } \\
\text { Adesão ao tratamento }\end{array}$ \\
\hline $\begin{array}{l}\text { The impact of illness perception } \\
\text { on adherence to therapeutic } \\
\text { regimens of patients with } \\
\text { hypertension in Taiwan. }{ }^{(9)}\end{array}$ & 2008 & $\begin{array}{l}\text { Analisar os efeitos da percepção da } \\
\text { doença sobre a adesão ao regime } \\
\text { terapêutico de pacientes com } \\
\text { hipertensão. }\end{array}$ & $\begin{array}{l}\text { Estudo } \\
\text { descritivo e } \\
\text { transversal }\end{array}$ & IPQ -R & $\begin{array}{l}\text { Educação em saúde } \\
\text { Adesão ao tratamento }\end{array}$ \\
\hline $\begin{array}{l}\text { Percepção sobre a hipertensão } \\
\text { arterial e qualidade de vida: } \\
\text { contribuição para o cuidado de } \\
\text { enfermagem. }{ }^{(10)}\end{array}$ & 2007 & $\begin{array}{l}\text { Descrever a percepção da doença } \\
\text { em pacientes hipertensos e sua } \\
\text { interferência na qualidade de vida. }\end{array}$ & $\begin{array}{l}\text { Estudo } \\
\text { descritivo } \\
\text { transversal }\end{array}$ & $\begin{array}{l}\text { Entrevista semi- } \\
\text { estruturada }\end{array}$ & $\begin{array}{l}\text { Educação em saúde } \\
\text { Adesão ao tratamento } \\
\text { Qualidade de vida }\end{array}$ \\
\hline $\begin{array}{l}\text { Qualidade de vida e percepção } \\
\text { da doença entre portadores de } \\
\text { hipertensão arterial. }{ }^{(11)}\end{array}$ & 2002 & $\begin{array}{l}\text { Analisar e associar a qualidade de } \\
\text { vida e percepção da gravidade da } \\
\text { doença em pacientes hipertensos. }\end{array}$ & $\begin{array}{l}\text { Estudo } \\
\text { observacional } \\
\text { transversal }\end{array}$ & $\begin{array}{l}\text { Entrevista semi- } \\
\text { estruturada }\end{array}$ & $\begin{array}{l}\text { Educação em saúde } \\
\text { Adesão ao tratamento } \\
\text { Enfrentamento } \\
\text { Qualidade de vida }\end{array}$ \\
\hline $\begin{array}{l}\text { Adultos hipertensos } \\
\text { hospitalizados: percepção } \\
\text { de gravidade da doença e da } \\
\text { qualidade de vida. }{ }^{(12)}\end{array}$ & 2001 & $\begin{array}{l}\text { Identificar a percepção da doença } \\
\text { e da qualidade de vida em adultos } \\
\text { hipertensos hospitalizados. }\end{array}$ & $\begin{array}{l}\text { Estudo } \\
\text { descritivo, } \\
\text { transversal }\end{array}$ & $\begin{array}{l}\text { Entrevista semi- } \\
\text { estruturada }\end{array}$ & $\begin{array}{l}\text { Educação em saúde } \\
\text { Adesão ao tratamento } \\
\text { Qualidade de vida }\end{array}$ \\
\hline
\end{tabular}

Quadro 1 - Descrição dos dados dos artigos incluídos no estudo

da saúde e o paciente, bem como ao próprio paciente.

O conhecimento disponível sobre a doença e o seu tratamento, sua percepção e eventual preocupação em relação ao diagnóstico, prognóstico, evolução, tempo de duração, gravidade da doença, bem como as experiências repetidas do paciente com hospitalizações são também fatores de extrema importância na adesão ao tratamento ${ }^{(14)}$.

A educação em saúde realizada pelos enfermeiros é responsável por criar condições de melhora na percepção e compreensão da doença por parte do paciente e assim, gerar o aumento da adesão ao regime terapêutico ${ }^{(11,12)}$.

A análise de como os indivíduos percebem sua doença pode auxiliar os profissionais da saúde a individualizar estratégias de prevenção secundária, melhorando assim, a adesão aos regimes terapêuticos. As discussões entre enfermeiro e paciente podem ter início com a identificação das variações na percepção que o paciente possui sobre sua doença ${ }^{(7,9,10)}$.

O comportamento dos pacientes em não aderirem ao tratamento é relevante, e sugere a necessidade de compreensão de seus valores, crenças e situação socioeconômica para que assim, os profissionais de saúde, em especial os enfermeiros possam agir juntamente ao paciente, conscientizando-o sobre a necessidade de adesão ao tratamento e ao mesmo tempo, fazê-lo sentir-se agente de mudança para melhorar e manter sua saúde ${ }^{(4,8)}$.

\section{Enfrentamento}

O enfrentamento (coping) é concebido como o conjunto das estratégias utilizadas pelas pessoas para se adaptar a circunstâncias adversas. Os esforços despendidos pelos indivíduos para lidar com situações estressantes, crônicas ou agudas, têm se constituído em objeto de estudo da psicologia social, clínica e da personalidade, encontrando-se fortemente atrelado ao estudo das diferenças individuais ${ }^{(15)}$.

O enfrentamento aparece como uns dos diagnósticos de enfermagem classificados pela NANDA Internacional (NANDA-I). Segundo essa classificação, é possível diagnosticar o enfrentamento levando em consideração sua deficiência e condições de melhora. Os diagnósticos encontrados para esta 
categoria compreendem o comprometimento e a ineficácia do enfrentamento pessoal e familiar bem como a disposição para o aumento do enfrentamento pelo paciente e pela família.

A melhora na percepção da doença é capaz de gerar no indivíduo resultados positivos em relação a sua capacidade funcional, desempenho físico, aspectos emocionais, aspectos sociais e evolução do estado de saúde ${ }^{(4,5,11)}$. Além disso, a melhora na percepção sobre a doença pode, ao longo do tempo, desencadear no paciente e em sua família a disposição para a confiança em si e nos outros ${ }^{(7)}$. Com isso é possível que o paciente desenvolva a capacidade de autocontrole, o que por sua vez permite maior adesão ao tratamento e eficácia em sua reabilitação ${ }^{(4,6,7)}$.

\section{Qualidade de vida}

A expressão "Qualidade de vida" é definida pela Organização Mundial da Saúde (OMS) como "a percepção do indivíduo de sua posição na vida no contexto da cultura e sistema de valores nos quais ele vive e em relação aos seus objetivos, expectativas, padrões e preocupações"(16). Qualidade de vida envolve o bem físico, mental, psicológico e emocional, além de relacionamentos sociais, como família e amigos e também a saúde, educação, poder de compra e outras circunstâncias da vida. A percepção que o paciente possui sobre a doença influencia em sua qualidade de vida, interferindo em suas condições de saúde e em outros aspectos da vida pessoal ${ }^{(11)}$.

A educação e intervenção por parte da enfermagem contribui para o aumento da adesão ao tratamento, promoção à saúde e adoção de um estilo de vida que reduza os fatores de risco, sintomas negativos e contribua para uma melhora da qualidade de vida ${ }^{(12)}$.

Para muitos pacientes, uma boa qualidade de vida significa boa saúde, boa relação familiar, lazer, alegrias, amizades e condições de moradia adequadas. Além disso, grande parte dos paciente apontam dificuldades em relação ao trabalho e mudanças nos hábitos de vida como as principais interferências da doença na qualidade de vida ${ }^{(10)}$.

As crenças negativas a respeito da doença são, na maioria das vezes, associadas a diminuição da qualidade de vida. Incentivar e apoiar os pacientes a aumentar a percepção que possuem a respeito de sua patologia pode ser um boa estratégia da equipe de enfermagem no programa de reabilitação(6).

\section{DISCUSSÃO}

Após a análise e interpretação dos dados da pesquisa primária e da categorização dos artigos, foi possível sintetizar e discutir os dados encontrados nos estudos.

Ao avaliar a percepção da doença, o enfermeiro tem a possibilidade de entender a maneira de o paciente lidar com a doença e recebe os subsídios necessários para a criação do plano de cuidado individualizado, levando em consideração os pontos que devem receber mais ou menos atenção.

Com um plano de cuidado bem orientado e direcionado às principais necessidades do paciente, o enfermeiro auxilia no reforço ou mudança da percepção do paciente em relação à doença, promovendo a saúde deste indivíduo ${ }^{(6-12)}$.
A melhora na percepção da doença pode permitir ao paciente maior disponibilidade para o aumento de outro fator relacionado a essa percepção, o enfrentamento. Compreender melhor sua patologia, seus efeitos, consequências e chances de cura faz com que o paciente encontre formas mais eficazes para tolerar e enfrentar sua doença $\mathrm{a}^{(5-7,11)}$.

Os enfermeiros podem notar a melhora do enfrentamento do paciente por meio de avaliações periódicas, utilizando as classificações dos diagnósticos de enfermagem da NANDA e os resultados de enfermagem (Nursing Outcomes).

A mudança de um diagnóstico de "Enfrentamento ineficaz" para o diagnóstico de "Disposição para enfrentamento aumentado" é o melhor resultado que pode ser encontrado em relação ao enfrentamento e percepção da doença. Quando o paciente passa a perceber melhor sua doença e aumenta seu enfrentamento em relação a esta, a chance deste aderir melhor ao tratamento também aumenta ${ }^{(4,11)}$.

A partir do momento em que o enfermeiro detecta os problemas e intervém na educação, o paciente torna-se conhecedor da patologia, seu tratamento, efeitos colaterais e consequências da não adesão ao regime terapêutico. Desta forma, o paciente é capaz de relacionar os efeitos contrários das medicações com a patologia e com isso, encontrar junto ao enfermeiro maneiras de amenizar a ocorrência e duração destes efeitos, tornando-se mais adeptos tanto ao tratamento farmacológico quanto o não farmacológico ${ }^{(4,7-12)}$.

Em relação à qualidade de vida, com a melhora da percepção sobre a doença, melhora da educação, enfrentamento e adesão ao tratamento, o indivíduo pode perceber uma melhora na qualidade de vida ${ }^{(6,10-12)}$.

Segundo os artigos que falam sobre este assunto, os pacientes dizem que para se ter uma boa qualidade de vida é necessário ter, não só boa saúde, mas também estar feliz em relação a si e aos outros ${ }^{(10,11)}$. Em alguns estudos aqui apresentados, os enfermeiros utilizaram instrumentos validados para avaliar a percepção da doença.

O Illness Perception Questionnaire (IPQ) é um método composto por 38 itens avaliados através de uma escala que varia de zero a cinco. Os itens compreendem a identidade da doença através dos sintomas, causas que o paciente aponta como responsáveis pela patologia, duração percebida da doença, consequências e os efeitos e resultados esperados pelo controle e cura ${ }^{(17)}$. Sua versão revisada (IPQ-R) possui mais de 80 itens e por ser um questionário longo muitas vezes não é indicado, principalmente quando o paciente está muito debilitado e é incapaz de respondê-lo inteiramente ou quando não há tempo disponível para o preenchimento de todos os itens ${ }^{(18,19)}$. Devido a isso, uma versão breve do questionário foi criada, a fim de diminuir o tempo de avaliação e melhorar o entendimento por parte dos avaliados. Essa versão é conhecida como Brief Illness Perception Questionnaire (B-IPQ).

O B-IPQ é constituído por nove itens que foram desenvolvidos através de uma questão capaz de resumir os itens contidos no IPQ-R. Estes itens por sua vez, são avaliados por meio de uma escala que varia de zero a dez. O B-IPQ além de válido e confiável, oferece aos pacientes completa e fácil compreensão, além dos resultados das escalas serem facilmente 
marcados e interpretados pelos pesquisadores, sendo aplicado em pacientes com doenças cardiológicas ${ }^{(20)}$.

O Illness Perception Questionnaire (IPQ) oferece o suporte necessário para os enfermeiros avaliarem a percepção da doença, a partir da ótica de quem a tem. A forma breve do questionário (B-IPQ) permite ao enfermeiro o conhecimento da percepção da doença em poucos minutos e de forma objetiva. A utilização deste questionário deve ser feita pelos enfermeiros desde o momento da admissão do paciente na unidade de internação ou local de atendimento e com os dados obtidos, traçar um plano de cuidado individualizado e com metas a serem alcançadas.

Além disso, a avaliação da percepção sobre a doença deve ser realizada periodicamente durante todo o tratamento do paciente, pois permite ao profissional perceber se há necessidades de mudanças do plano de cuidado e com isso, proporcionar uma reabilitação mais rápida e eficaz.

\section{CONCLUSÕES}

Avaliar a percepção da doença é uma tarefa que exige atenção e perspicácia do profissional, que deve conhecer o nível de informações que o paciente possui sobre sua doença, bem como as reações e sentimentos presentes em razão da patologia.

Os artigos estudados mostram a necessidade em se avaliar a percepção da doença e a importância da equipe de enfermagem nesta análise para que assim, sejam propostas uma série de intervenções através de um plano de cuidado individualizado que promova a melhora na educação, adesão ao regime terapêutico, aumento do enfrentamento e reabilitação do doente.

É necessário que os enfermeiros passem a utilizar periodicamente instrumentos para a avaliação da percepção da doença como por exemplo, o B-IPQ, além do uso rotineiro dos diagnósticos de enfermagem que no Brasil, ainda não são utilizados por todos os enfermeiros e instituições.

\section{REFERÊNCIAS}

1. Reis J, Fradique F. Desenvolvimento sociocognitivo de significações leigas em adultos: causas e prevenção das doenças. Anál. Psicol. 2002 jan;20(1):5-26.

2. Figueiras MJ, Machado V, Alves NC. Os modelos de senso-comum das cefaléias crônicas nos casais: relação com o ajustamento marital. Anál. Psicol. 2002; jan 20(1):70-90.

3. Sampieri RH, Collado CF, Lucio PB. Capítulo 10: Análisis de los datos. Metodología de d investigación. $2^{\mathrm{a}} \mathrm{d}$. México: McGraw-Hill; 1998. p. 579-607.

4. Chen SL, Tsai JC, Chou KR. Illness perceptions and adherence to therapeutic regimens among patients with hypertension: A structural modeling approach. Int. J. Nurs. Stud. 2011; fev 48(2):235-45.

5. Yan J, You LM, He JG, Wang JF, Chen L, Liu BL et al. Illness perception among Chinese patients with acute myocardial infarction. Patient. Educ. Couns. 2011 Dec 85(3):398-405.

6. Alsén $P$, Brink E, Persson LO, Brändström $Y$, Karlson BW. Illness Perceptions After Myocardial Infarction: Relations to Fatigue, Emotional Distress, and Health-Related Quality of Life. J. Cardiovasc. Nurs. 2010 Mar/Apr;25(2): 1-10.

7. Alsén P, Brink E, Persson LO. Patients' illness perception four months after a myocardial infarction. J. Clin. Nurs. 2008;17(5A):25-33.

8. Link $\mathrm{Cl}$, Bielemann VLM, et al. Paciente crônico frente ao adoecer e a aderência ao tratamento. Acta Paul. Enferm. 2008;21(2):317-22.

9. Chen SL, Tsai JC, Lee WL. The impact of illness perception on adherence to therapeutic regimens of patients with hypertension in Taiwan. J. Clin. Nurs. 2009 Aug; 18:2234-44.

10. Pinotti S, Mantovani MF, Giacomozzi LM. Percepção sobre a hipertensão arterial e qualidade de vida: contribuição para o cuidado de enfermagem. Cogitare Enferm. 2007; 13(4):526-34.
11. Brito DMS, Araujo TL, L L. Qualidade de vida e percepção da doença entre portadores de hipertensão arterial. Cad. Saúde Pública 2002;24(4):933-40.

12. Reis MG, Glashan RQ. Adultos hipertensos hospitalizados: percepção de gravidade da doença e da qualidade de vida. Rev. Latino-Am Enfermagem 2001 maio;9(3):51-7.

13. Campos JADB, Zuanon ACC, Guimarães MS. Educação em saúde na adolescência. Cienc. Odontol. Bras. 2003 out/dez;6(4):48-53.

14. Dewulf NLS. Investigação sobre a adesão ao tratamento medicamentoso em pacientes com doenças inflamatórias [dissertação]. Ribeirão Preto (SP): Faculdade de Medicina de Ribeirão Preto, Universidade de São Paulo; 2005.

15. Andra F. A atividade física e o enfrentamento de estresse em idosos [dissertação]. São Paulo (SP): Faculdade de Medicina, Universidade de São Paulo; 2010.

16. Departamento de Psiquiatria da Faculdade de Medicina da UFRGS. Versão em português dos instrumentos de avaliação de qualidade de vida (WHOQOL). Rio Grande do Sul; 2003. [citado em 17 jul 2010]. Disponível em: http://www.ufrgs.br/psiq/whoqol.html

17. Weinman J, Petrie KJ, Moss-Moris R, Horne R. The Illness Perceptions Questionnaire: a new method for assessing the cognitive representation of illness. Psychology and Health 1996;11:431-45.

18. Moss-Moris R, Weinman J, Petrie K, Horne R, Cameron L, Buick D. The revised Illness Perception Questionnaire (IPQ-R). Psychology and Health 2002;17: 1-16.

19. Figueiras MJ, Alves NC. Lay perceptions of serious illnesses: An adapted version of the Revised IIIness Perception Questionnaire (IPQ-R) for healthy people. Psychology and Health 2007; 22:143-158.

20. Broadbent E, Petrie KJ, Main J; Weinman J. The Brief Illness Perception Questionnaire. J. Psychosom. Res. 2006;60:631-637. 